\title{
Potential Benefits of Ubiquinone in Patients with Statin Induced Myalgia
}

\section{Emil Ștefănescu'1,2*}

1"Carol Davila" University of Medicine and Pharmacy, Faculty of Pharmacy, Department of Pharmacology and Clinical Pharmacy, Bucharest, Romania

2"Tehnoplus Farm Ltd" Community Pharmacy, Romania

*Corresponding Author: Emil Ștefănescu, "Carol Davila” University of Medicine and Pharmacy, Faculty of Pharmacy, Department of Pharmacology and Clinical Pharmacy, Bucharest, Romania.

Received: September 11, 2019; Published: September 20, 2019

DOI: $10.31080 /$ ASPS.2019.03.0400

\section{Abstract}

Coenzyme Q-10 is well known for its role in cellular respiration, antioxidant properties and pro-inflammatory gene expression. Q-10 blood levels naturally decrees with age, but also in patients treated with cholesterol lowering statin drugs. Consequently myalgia and myophaty tend to occur. Patients reported improved muscular condition after administering ubiquinone dietary supplements containing ubiquinone.

Keywords: Ubiquinone; Statin; Myalgia

\section{Introduction}

Cholesterol lowering statin drugs often cause muscular destruction [1]. This side effect appears to be related to the decrease of ubiquinone blood and muscular levels which occur in patients treated with statins because cholesterol and ubiquinone share the same synthesis rout [2].

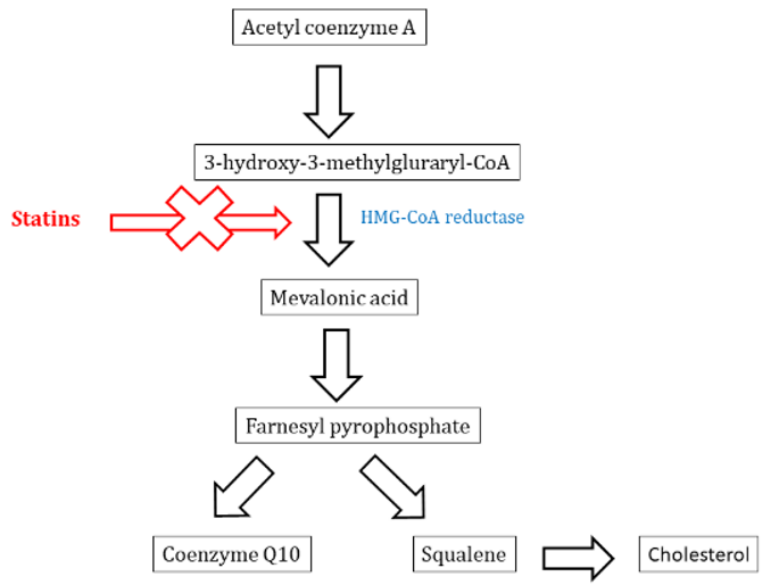

Figure 1

Some studies show that increasing exogenous intake of ubiquinone in order to maintain normal blood and muscular levels may contribute to lowering the muscular side effect risks in patients treated with statins for high blood cholesterol [3].

\section{Case Presentation}

Three patients ( 2 women - age 75 and 1 man - age 79) with high blood pressure and high blood cholesterol levels, undergoing long term treatments with simvastatin $40 \mathrm{mg} /$ day, complained to the local pharmacist of muscular pain in the legs and difficulty walking. The pharmacist reported this complains to the prescribing physicians and was told that the benefit - risk ratio is positive for all 3 patients despite the side effects. Knowing the connection between ubiquinone and cholesterol synthesis as well as the statin mechanism of action, the pharmacist recommended all 3 patients a dietary supplement containing Coenzyme Q-10 to be administered daily in a $200 \mathrm{mg} /$ day dosage alongside the statin prescribed by the physicians.

\section{Results and Discussion}

After 3 month of administering simvastatin $40 \mathrm{mg} /$ day and ubiquinone $200 \mathrm{mg} /$ day, one of the women reported to the pharmacist a complete remission of the muscular symptoms, and the other one indicated a significant improvement. After 4 month of administering the same combination, the male patient also reported a significant improvement in the muscular symptoms.

\section{Conclusion}

The pharmacist can play a major role not only in identifying and reporting drug side effects observed in patients, but also in presenting viable therapeutic options for neutralizing them. 


\section{Bibliography}

1. Hilton-Jones D. "Statin-related myopathies". Practice Neurology 18.2 (2018): 97-105.

2. Passi S., et al. "Statins lower plasma and lymphocyte ubiquinol/ubiquinone without affecting other antioxidants and PUFA". Biofactors 18.1-4 (2003): 113-124.

3. Tan JT and Barry AR. "Coenzyme Q10 supplementation in the management of statin-associated myalgia”. American Journal of Health-System Pharmacy 74.11 (2017): 786-793.

Volume 3 Issue 10 October 2019

(C) All rights are reserved by Emil Ștefănescu. 